\title{
Dynamic Arrays Based on Magnetically Controlled Particles: Synthesis and Application
}

\author{
Ivan Shorstkii **i] \\ ${ }^{a}$ Kuban State University of Technology, Department of Technological equipment and life-support systems, \\ Krasnaya Street 135, Krasnodar, Russian Federation
}

Received: April 28, 2018; Revised: April 09, 2019; Accepted: June 29, 2019

\begin{abstract}
Growth mechanisms of dynamic granular arrays based on magnetically controlled particles are presented. Method of the external rotating magnetic field (ERMF), based on a dipole interaction of magnetic spherical $\mathrm{Fe}_{3} \mathrm{O}_{4}$ particles in highly ordered volume arrays is proposed and a design for its practical implementation is developed. The magnetic assembly can result in permanent structures, which could be disassembled on demand by remote demagnetization. To study the interaction field of particles in the arrays, a mean field theory was used and the data were compared with experiment. Reflection spectra of electromagnetic radiation from a volume dense packed array of $\mathrm{Fe}_{3} \mathrm{O}_{4}$ particles with thicknesses of 3 and $6 \mathrm{~mm}$, and a flat particles array with a set of 15 and 30 layers, as well as frequency characteristics of attenuation of electromagnetic radiation of the claimed materials in the frequency range from $8.0 \mathrm{GHz}$ to $12.5 \mathrm{GHz}$ obtained. Presented method and the installation have the prospect of being used in the processes of making composite materials for electromagnetic radiation protection using a wide range of materials of micro and nanoparticles.
\end{abstract}

Keywords: magnetically controlled particles, rotating magnetic field, array, composite material, microwave adsorption.

\section{Introduction}

Arising from the rapid development of electronic devices, wireless communication tools, and local area networks, electromagnetic interference (EMI) has greatly threatened human health and disturbed various commercial or industrial equipment ${ }^{1-2}$. Thus, the demand for electromagnetic absorbents has become urgent over the past years. It is well-known that an electromagnetic absorbent is a type of functional material that is able to absorb incidence of electromagnetic wave effectively and then convert it into thermal energy. The ideal absorbing materials must have light weight, strong absorption, thin thickness, and broad frequency. Several factors, such as morphology, geometry and microstructure, are crucial in determining the electromagnetic absorption properties. Currently, different morphologies of absorbents have been fabricated by various methods, containing onedimensional (1-D; nanowires, nanoparticles), twodimensional (2-D; flake, nanodisks) and three-dimensional (3-D; tubes, sphere, urchin-like structure $)^{3-5}$.

In this study, a novel method was designed for porous 3-D granular arrays fabrication based on magnetically controlled particles as a new type of microwave absorption materials. These well-defined structures consist of numerous air pores. At the same time, the porous structure with a lower density can be used as lightweight microwave absorption material. Method of external rotating magnetic field (ERMF), based on a dipole interaction of magnetic spherical $\mathrm{Fe}_{3} \mathrm{O}_{4}$ particles in highly ordered volume arrays is proposed to form the array of particles.
When magnetic particles are placed inside the ERMF the particles are aligned along the field lines of the dipole field and are entrained in its displacement ${ }^{6}$. On the basis of this principle, a technique is developed for the formation of dynamic flat and 3-D particles arrays with a given packing structure.

\section{Experimental Section}

$\mathrm{Fe}_{3} \mathrm{O}_{4}$ microparticles were purchased from Ricoh Company (Japan). Particles composition were determined by X-ray phase analysis (XRD) on a Shimadzu XRD-7000 $\mathrm{S}$ diffractometer (Japan).

The 3-D composite was of dynamic arrays prepared using external rotating magnetic field effect on lab setup (Fig. 1). Experimental lab setup consists of a platform, cylindrical tube, nozzle with permanent magnets and two stepper motors with a control panel.

On the outside of the tube, 1 is a cylindrical nozzle 2 with diametrically opposed two permanent magnets 3 disposed of therein and a mechanical pole switcher 4 . The cylindrical nozzle has a gear train 5 through which it is connected to a gear 6 connected to an electric motor 7. Inside tube, there are $\mathrm{Fe}_{3} \mathrm{O}_{4}$ particles. Neodymium magnets create an external rotating magnetic field around the tube and fixed in the nozzle and closely contact with a glass tube.

Particles placed inside the tube and captured by the magnetic field of permanent magnets 3. Cylindrical nozzle 2 starts rotating and reconstructing particles from the fibrous dispersed into a dense package, forming particles array ${ }^{7-8}$. 
When magnetic particles placed into a permanent magnetic field, the particles tend to line up and oriente themselves along the magnetic field lines (Fig. 2). When imposing ERMF on an array of particles located in the tube, a change in their structure occurs. Particles movement is a closed trajectory resembling the "Figure 8 ". It is worth noting the delayed movement of magnetic particles relative to the central axis of the permanent magnets in the direction of rotation by $10-12^{\circ}$. This is due to the release of individual particles from the array chain and their integration into the adjacent chain during mutual dipole interaction. With an increase in the angle of rotation of the main vessel, the trajectory of motion of the particles remains, however, more particles begin to be involved in the movement.
At an angle of rotation of $180^{\circ}$, the number of fibrous structures is reduced, and the surface of the particle array acquires a more densely packed structure. As the experiment showed, one turn of the main vessel is enough to form an array with a uniform density throughout the volume and a cubic facecentered dense packing of particles.

To obtain a composite material and study reflection spectrum and the frequency characteristics of attenuation of electromagnetic radiation, paraffin was added to the medium of the particle array in a volume ratio of $1: 1$. This composition was brought to the melting point of the paraffin with an alcohol burner. After a homogeneous mass was obtained, the tube was moved to the zone of the magnetic packing by means of a reciprocating platform.
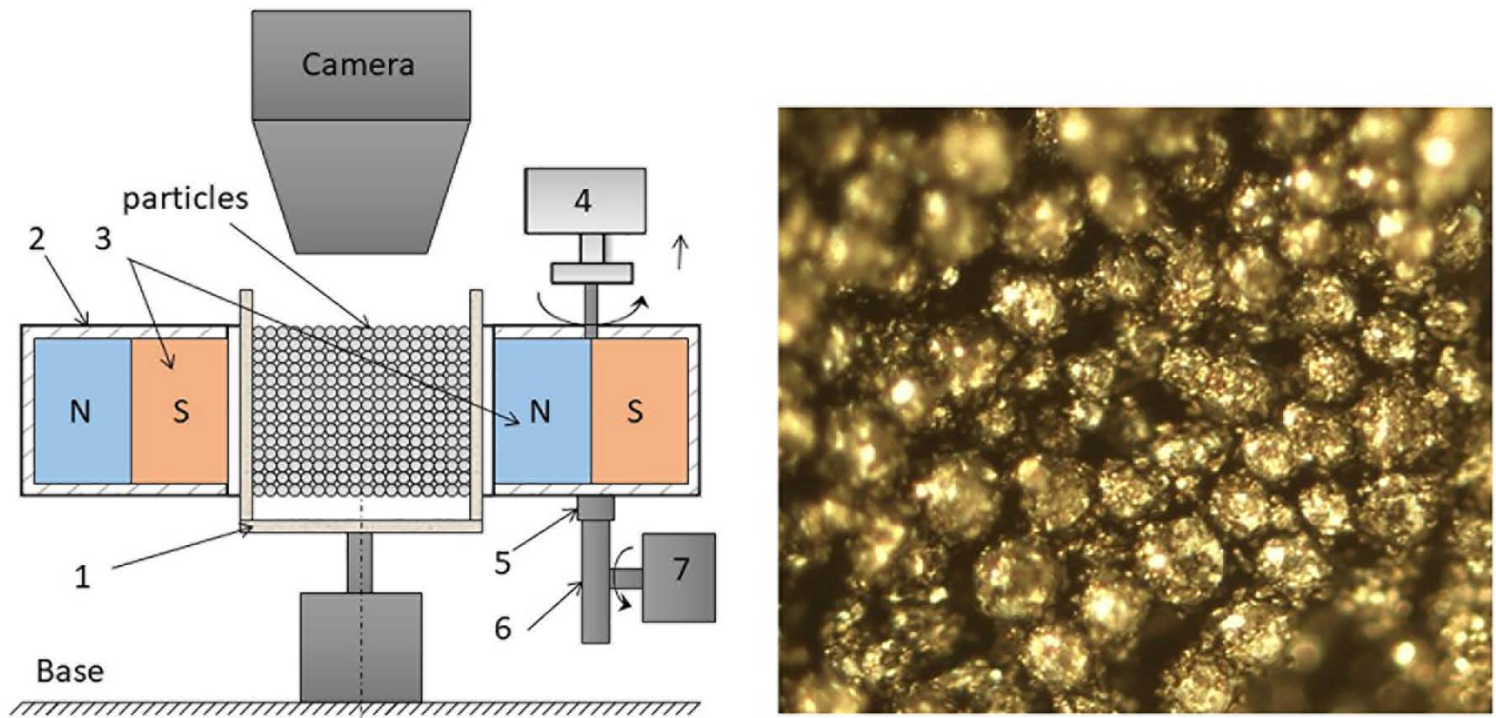

Figure 1. ERMF lab setup and flat sample.
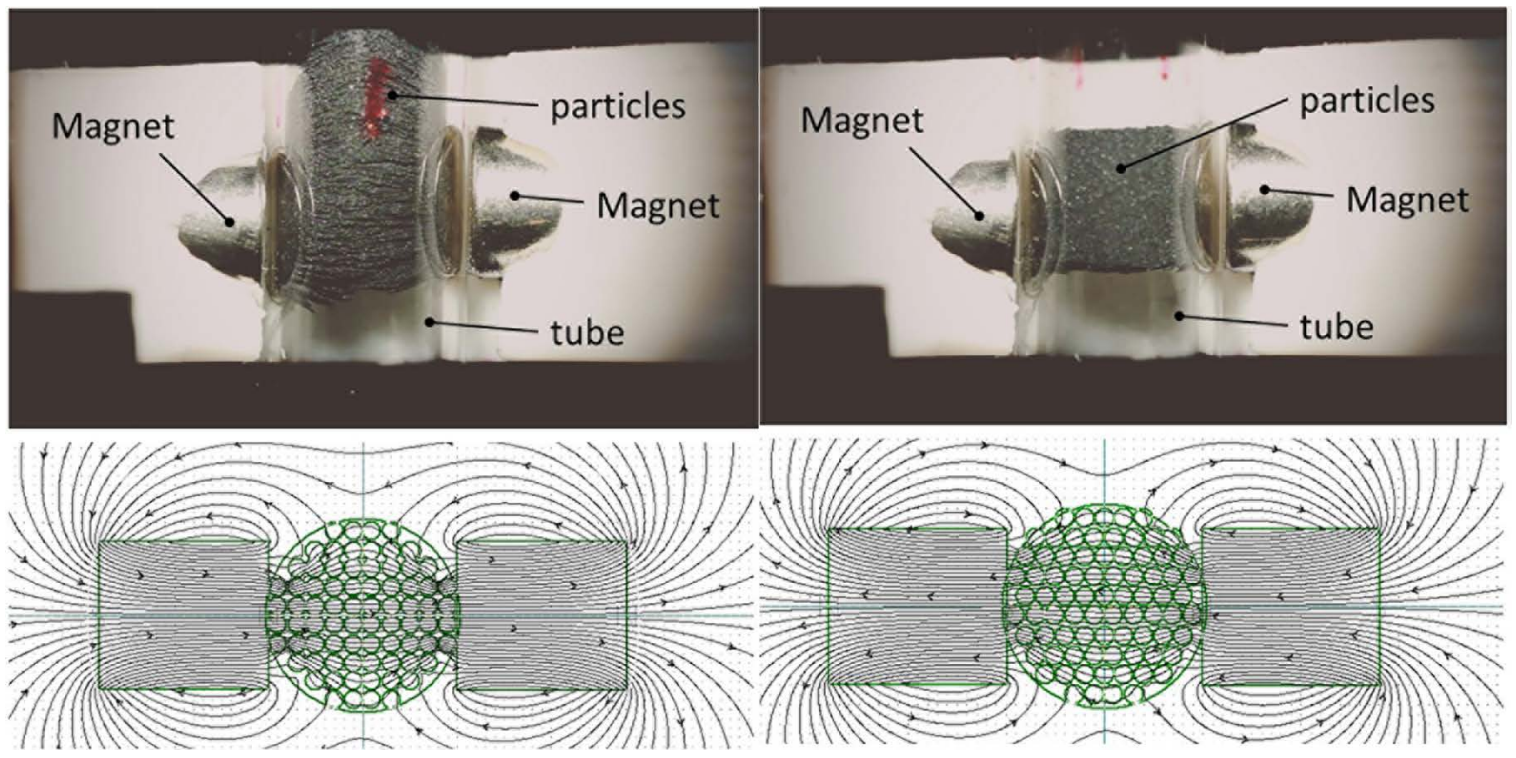

Figure 2. Particles in a tube at initial position (left) holding by permanent magnets with vizimag simulation of particles array structure and particles in a tube after rotation of permanent magnets (right) with a vizimag simulation of particles array structure. 
Permanent magnets provided the creation of an external rotating magnetic field around the tube, to form dynamic particles arrays. After the paraffin had solidified, the composite was removed. Then the compact sample was cut into parallelepiped with $23.0 \mathrm{~mm} \pm 10.0 \mathrm{~mm} \pm 3.0 \mathrm{~mm}$ size. During samples preparation the length of samples was fit with the direction of magnetic field.

Sample with flat arrays (Fig. 1) was prepared by pressing a sticky tape against particles and reciprocating translational motion of magnetic dipole along the plane of a sticky tape, then place another sticky tape for sealing. To study individual particles, particles were separated by a needle and placed on sticky tape, forming dispersed arrays. As an extension to current research, samples with larger inter-array distance were made.

A time varying magnetic field was used to magnetize the samples on Vibrating Sample Magnetometry (VSM, IMRE, Singapore). Furthermore, the vibrating sample holder is secured on a rotation stage that allows variation of $\varphi$ between $0^{\circ}$ and $90^{\circ}$ in order to analyse magnetization of the sample at different azimuth angle $\varphi$ between external field and sample plane.

The composition and phase purity of samples were analyzed by X-ray diffraction (XRD) at $40.0 \mathrm{kV}$ voltage and $30.0 \mathrm{~mA}$ current with $\mathrm{CuK} \alpha$ radiation $(\mathrm{l}=1.541 \AA$ ). Scanning electron microscopy (SEM) images were obtained on a JEOL JSM 6300 scanning electron microscope at an acceleration voltage of $15.0 \mathrm{kV}$.

The microwave-absorbing characteristics were evaluated by measuring the reflection loss using an P2-61 scalar quantity network analyzer in the $8.0 \mathrm{GHz}$ to $12.0 \mathrm{GHz}$ band range. All the measurements were performed at room temperature.

The RL coefficient curves were calculated from the relative permeability and permittivity for a given frequency and absorber thickness, according to the following equations:

$$
R L(d B)=20 \log \left|\left(Z_{\text {in }}-Z_{0}\right) /\left(Z_{\text {in }}+Z_{0}\right)\right|
$$

where $Z_{0}$ - the impedance of free space and $Z_{\text {in }}$ - the input impedance of the absorber.

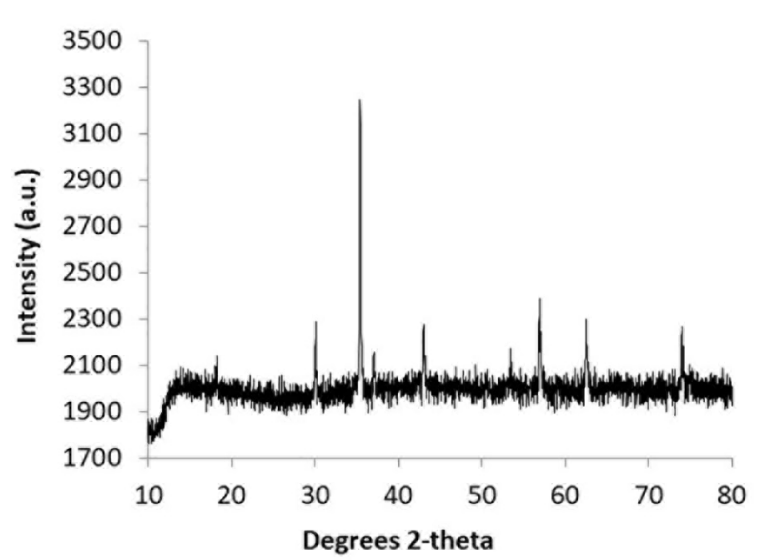

\section{Results and Discussion}

Figure 3 shows the XRD pattern of samples. Analysing the X-ray diffraction pattern of the investigated particle samples, it was found that a significant part of the substance is in the x-ray morphological state. When compared to the PDF-2 and PDF-4 database, the material under investigation was identified as Iron Oxide $\left(\mathrm{Fe}_{3} \mathrm{O}_{4}\right)$.

When the process of rotation of the nozzle with permanent magnets around the cylindrical tube occurs, the spherical particles $\mathrm{Fe}_{3} \mathrm{O}_{4}$ are densified with a uniform distribution of the particle density in the 3-D of the array (Fig. 3). A transition occurs from the dispersed fibrous structure of spherical particles to a dense package with a porosity $\varepsilon=0.259$ close to the porosity of a face-centred package.

Figure 3 is a low magnification SEM image showing a group of $\mathrm{Fe}_{3} \mathrm{O}_{4}$ particles. These particles are nearly spherical in shape with a narrow diameter distribution of around $50 \mu \mathrm{m}$ to $60 \mu \mathrm{m}$. The SEM image, as shown in Figure 3, clearly indicates that all the particles own a 'core/shell' type microstructure and the inner Fe cores are encapsulated into the 'onion' type carbon cages. The formed volume array of micro spherical particles has a porous channel structure with pores of approximately $15.0 \mu \mathrm{m}$ in diameter.

Magnetic anisotropy was more clearly observed in compact arrays-the dependence of parallel and perpendicular magnetization on the direction of the applied field with respect to the sample plane samples reach saturation slower and at higher field as $\varphi$ increases (Fig. 4). Since smaller distance between adjacent particles would entail interaction, was reasonably to postulate that interaction between particles may result in the anisotropy observed. Moreover, at the same $\varphi$, compact samples reach saturation faster than dispersed samples. Thus, if interaction does exist, it would most likely have intensifying effect on external field. The hypothesis can only be tested out through modeling of the interaction in compact array case within mean field approximation and compare it with the experimental data.

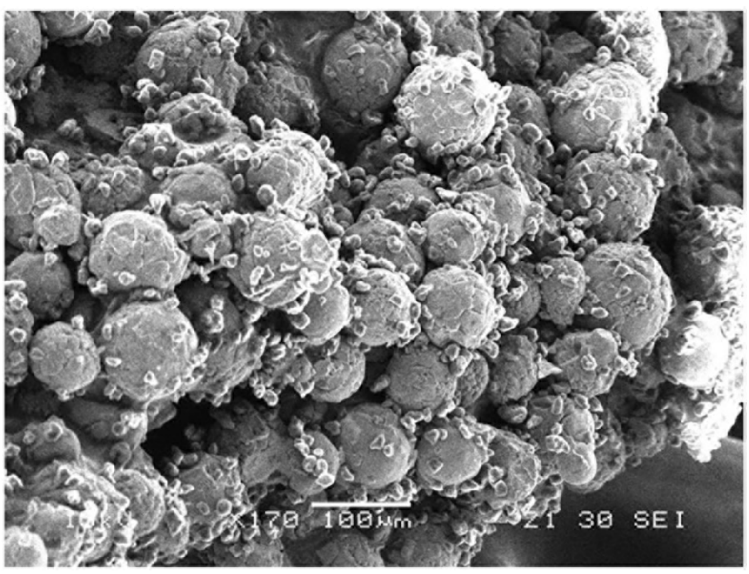

Figure 3. XRD patterns (left) and SEM images of 3-D particles arrays (right). 

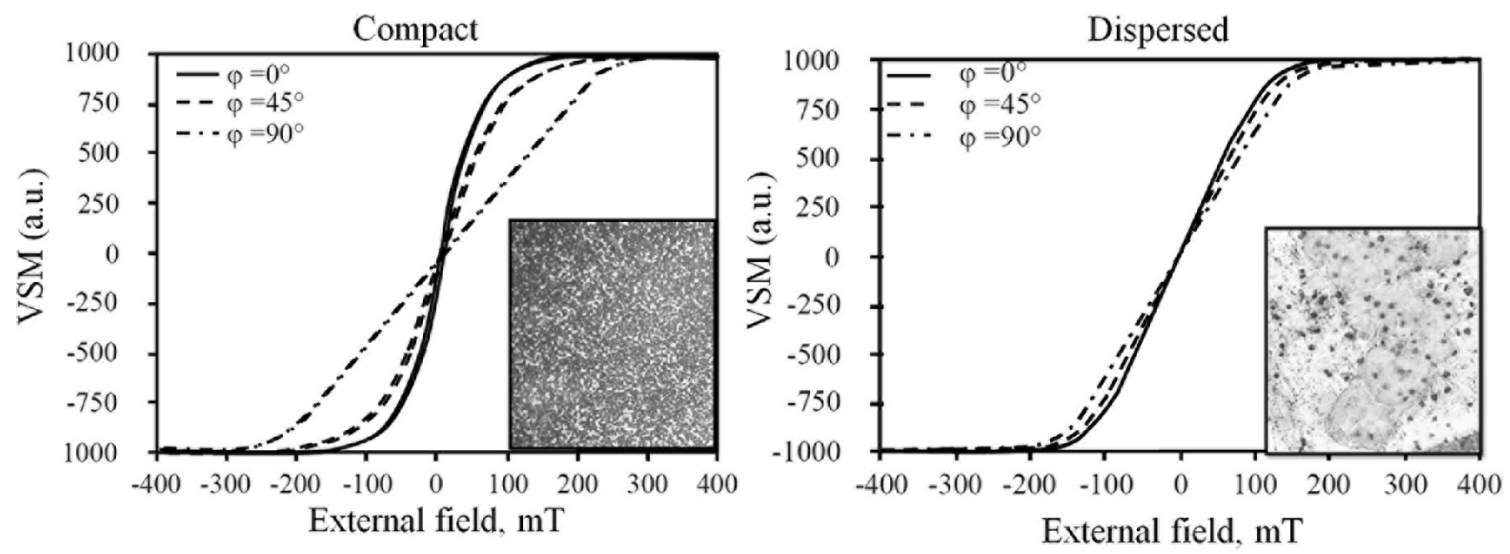

Figure 4. Magnetization curves for compact and dispersed packaging at azimuth $0^{\circ}, 45^{\circ}$ and $90^{\circ}$.

However, the magnetic moment density of the particles is required for applying mean field approximation and it can only be obtained from the dispersed arrays. As a result, dispersed arrays of both shape were first modeled to facilitate the modelling of interaction field in compact arrays.

For 2D arrangement where particles were dispersed by a distance of more than 10 times of their radius, interaction among particles was minimized. Thus, the entire $2 \mathrm{D}$ plane can be modeled by considering a single particle due to the relative uniformity of particle size. When external magnetic field is applied, randomly arranged atomic dipoles inside the particle slowly align with the external field. Due to isotropic property of spherical particle and the absence of magnetic dipole interaction among particles, the total magnetization of the selected particle is always parallel or antiparallel to the external field. The process of magnetization can be modeled by a domain wall with zero width that sweeps through the particle to increase the number of dipoles that align with the external field. As a reasonable simplification, particles are assumed to be cubic in shape and the domain wall is parallel to both external field and two sides of the cube.

At any external field, the domain wall position always minimizes the Zeeman Energy of the selected particle, which is the potential energy of the magnetized particle in an external magnetic field. By equating the first derivative of energy with respect to domain wall position to be zero, the expression of the domain wall position at any external field before saturation is found to be $y_{0}=b+\pi B_{e x t} \alpha / \mu_{0} M$. With domain wall position determined and the magnetization of the sample in terms of the external magnetic field expressed. The sample reaches saturation when $\mathrm{y}=\mathrm{a}$. Thus, the corner field at which the sample saturates is $B_{\text {cor }}=\mu_{0} M / \pi$. After saturation, the magnetization of the particle remains constant. By comparing the model curve and the experimental curve (Fig. 5) was found that our model is capable of accurately predicting the saturation rate and saturation point for $2 \mathrm{D}$ dispersed sample with spherical particles. Saturation magnetization is reached at around $120.0 \mathrm{mT}$ and the magnetic moment density is found to be around $3040 \mathrm{~A} / \mathrm{m}$.
Particles in compact sample are suspected to interact with each other. The expression of the interaction field can be derived with mean field approximation. A point at the edge of a semi-infinite plane is chosen and the field from surrounding particles is doubly integrated in polar coordinate from zero to infinity and zero to $2 \pi$. Distance between particles was first assumed to be zero for simplification purpose and was account for later by a parameter $a^{2} / d^{2}$ representing the density of the sample (Fig. 5).

Magnetization of the samples at field lower than saturation was plotted against $\varphi$ using formula?

$$
M_{\text {para }}=\frac{B_{\text {ext }}}{\mu_{0}} \cdot\left(\frac{\cos ^{2} \phi}{1-a^{2} / 2 D^{2}}+\frac{\sin ^{2} \phi}{1+a^{2} / D^{2}}\right)
$$

We derived and was compared with the experimental data (Fig. 6).

The interactions between particles were found to be surprisingly high compared to the average distance between particles, with calculated a/D ratio of 0.55 in dispersed samples, 0.95 in compact samples.

After saturation, further increment of external field will not result in changes in the magnitude but change in direction of total magnetization. This is because the alignment of magnetization direction with external field direction minimizes energy of the sample.

To further reveal the microwave absorption properties of the $\mathrm{Fe}_{3} \mathrm{O}_{4}$ composites, the reflection loss $\mathrm{R}$ was analyzed by comparing known literature data of $\mathrm{Fe}_{3} \mathrm{O}_{4}$ particles ${ }^{10-11}$ and obtained experimental data. A comparison of the reflection coefficient data presented as spectra are shown in Fig. 7. The maximum value of RL was obtained for a sample of a $3.0 \mathrm{~mm}$ thick volume array at a frequency of $12.0 \mathrm{GHz}$ and corresponded to $3.5 \mathrm{~dB}$. Increasing the thickness of the sample leads to an increase in the reflection coefficient. From the comparative analysis, it can be seen that the formed structure of densely packed particles has better electromagnetic fields protection properties in comparison with chaotically ordered particles. 


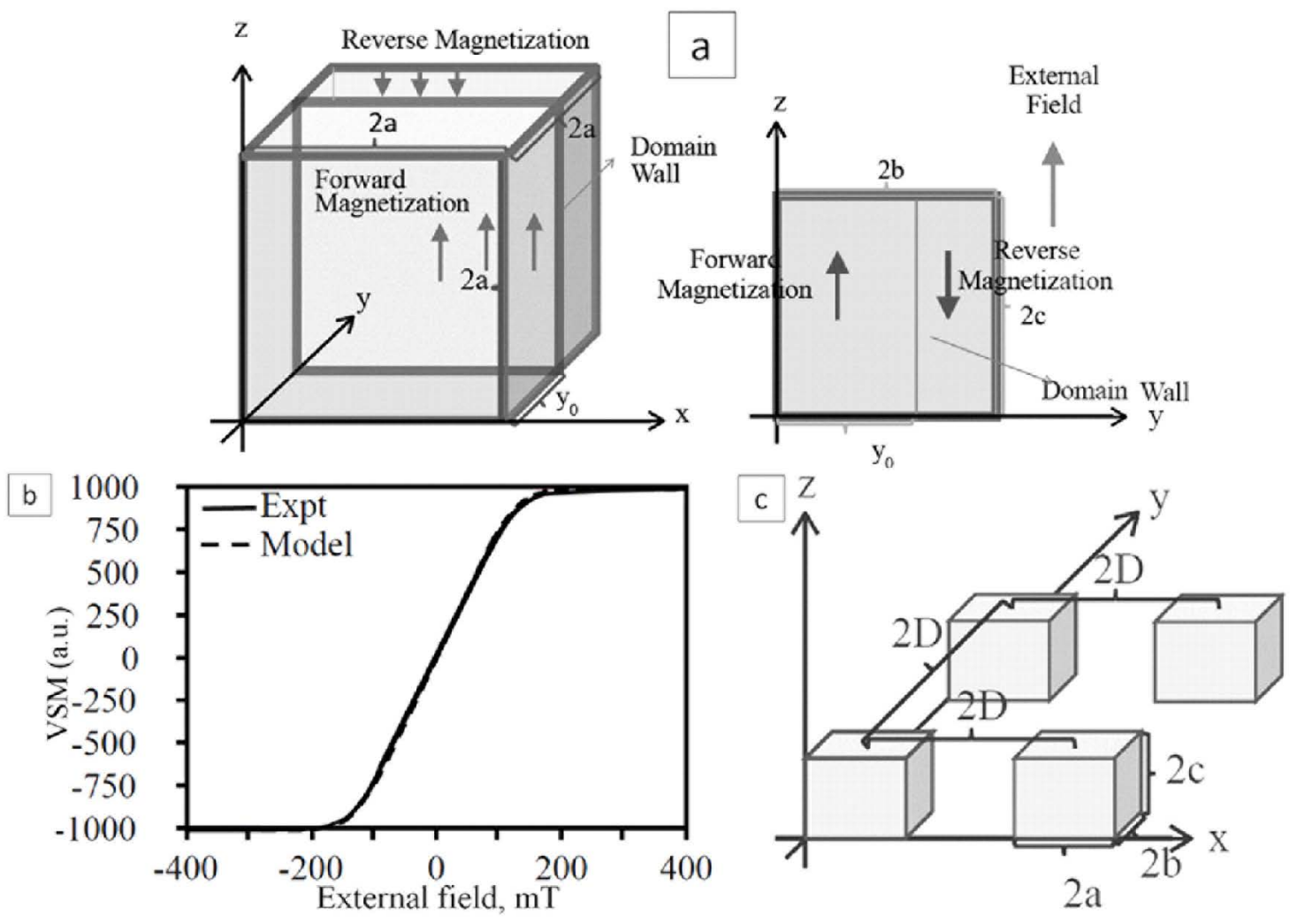

Figure 5. Schematic of domain wall position (a), comparison between model and experiment (b), and schematic of arrays on sample plane (c).
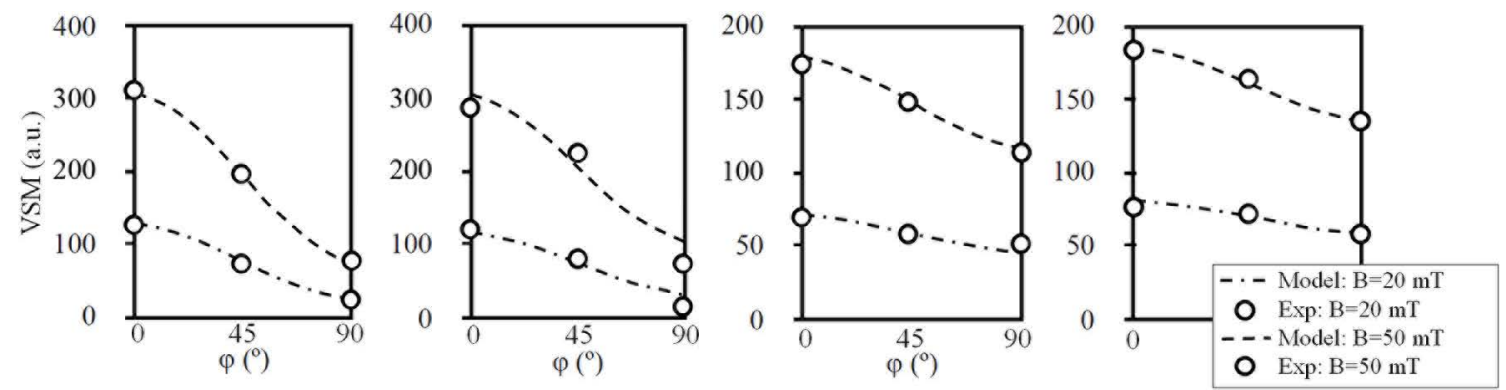

Figure 6. Plot of magnetization against azimuth angle $\varphi$.
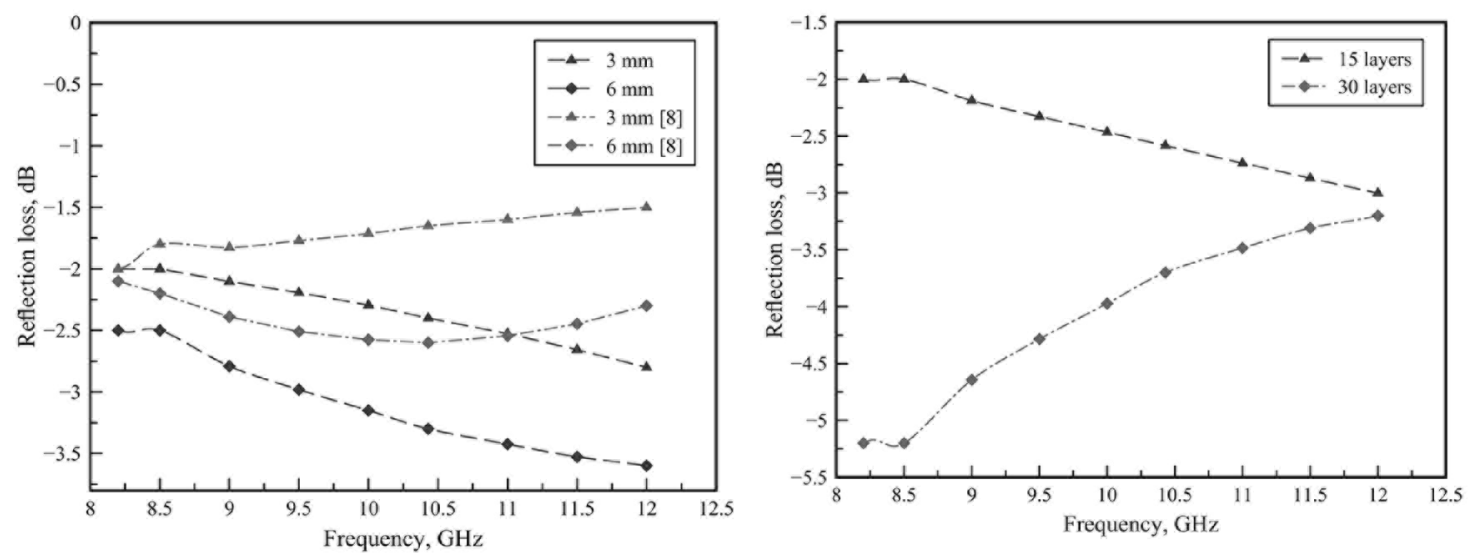

Figure 7. Microwave reflection loss $\mathrm{RL}_{\mathrm{L}} \mathrm{Fe}_{3} \mathrm{O}_{4}$ arrays particles-paraffin wax sample versus frequency and layer of flat arrays. 
The results of measuring the reflection loss for samples of flat arrays differing in the number of layers are shown in Fig. 6. The maximum RL value $-5.2 \mathrm{~dB}$ fixed at a frequency of 8.5GHz. Unlike the spectra of 3-D arrays, increasing the layers of flat arrays leads to a decrease in the reflection coefficient because of partial energy loss of electromagnetic radiation in interaction with a flat 2D array depends on the dipole-dipole interaction, and the surface density of $\mathrm{Fe}_{3} \mathrm{O}_{4}$ composite.

3-D samples thickness leads to double increase EMR attenuation coefficient from $13.0 \mathrm{~dB}$ for $3.0 \mathrm{~mm}$ sample up to $24.0 \mathrm{~dB}$ for a $6.0 \mathrm{~mm}$ sample in $(8 \ldots 12) \mathrm{GHz}$ range (Fig. 8). The increase of the samples thickness of flat arrays leads to double decrease the EMR attenuation coefficient from $14.0 \mathrm{~dB}$ for sample with 15 flat layers based on $\mathrm{Fe}_{3} \mathrm{O}_{4}$ particles up to $7.0 \mathrm{~dB}$ for the sample in with 30 layers at $12.0 \mathrm{GHz}$ frequency.

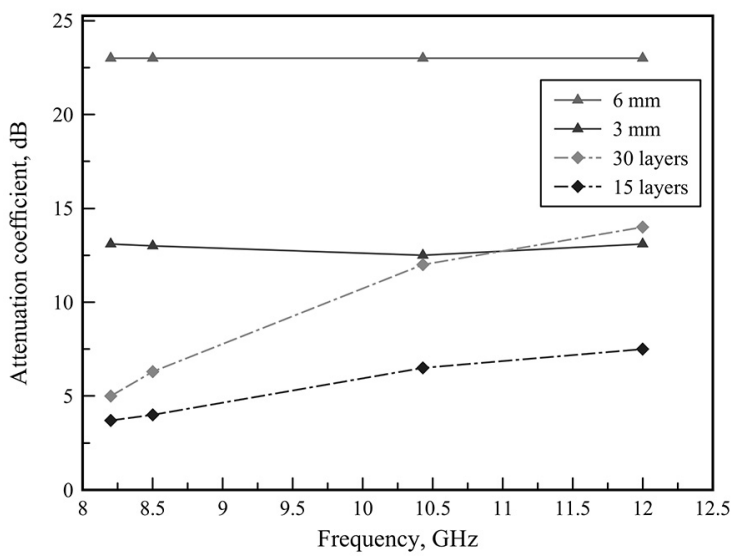

Figure 8. Attenuation coefficient of $\mathrm{Fe}_{3} \mathrm{O}_{4}$ arrays particles-paraffin wax sample and layer of flat arrays versus frequency.

\section{Conclusion}

The method of rotating magnetic field of dipoles and the installation for its realization have shown the possibility of forming dynamic 3-D arrays of spherical particles with porosity $\varepsilon=0.259$. Composite material obtained by this method has a small coercive force and a small residual magnetization, which affects the reflection spectra of the electromagnetic radiation. The magnetic susceptibility of the claimed composite material depends on the physical nature of the particles of the $\mathrm{Fe}_{3} \mathrm{O}_{4}$ material and on its geometric shape. Interaction between particles in compact arrays has been modelled within mean field approximation. Fitting to our data shows that the interactions between particles were found to be in good agreement with the average distance between particles. Part of the energy of electromagnetic radiation when interacting with this material is dissipated to overcome the demagnetizing factor associated with geometric parameters and to overcome the magnetic susceptibility associated with the nature of the material. Thus, the partial loss of the energy of electromagnetic radiation in interaction with a flat 2D array depends on the dipole-dipole interaction, and the surface density of the $\mathrm{Fe}_{3} \mathrm{O}_{4}$ composite.
By using rotational magnetic field of dipoles based on nano and microparticles interactions, it is possible to create types of microwave absorptive materials in a wide frequency range while maintaining strong absorption that have mechanical strength, manufacturability with usability.

\section{Acknowledgments}

The reported research was funded by Russian Foundation for Basic Research and the government of the region of the Russian Federation, grant No 19-42-233001.

\section{References}

1. Nazir A, Yu H, Wang L, Haroon M, Ullah RS, Fahad S, et al. Recent progress in the modification of carbon materials and their application in composites for electromagnetic interference shielding. Journal of Materials Science. 2018;53(12):8699-8719.

2. Liu J, Zhang HB, Sun R, Liu Y, Liu Z, Zhou A, et al. Hydrophobic, Flexible, and Lightweight MXene Foams for High-Performance Electromagnetic-Interference Shielding. Advanced Materials. 2017;29(38):1702367.

3. Liu Q, Cao Q, Bi H, Liang C, Yuan K, She W, et al. CoNi@ SiO2@TiO2 and CoNi@Air@TiO2 Microspheres with Strong Wideband Microwave Absorption. Advanced Materials. 2016;28(3):486-490.

4. Zhang XF, Dong XL, Huang H, Lv B, Lei JP, Choi CJ. Microstructure and microwave absorption properties of carboncoated iron nanocapsules. Journal of Physics D: Applied Physics. 2007;40(17):5383.

5. Ido Y, Li YH, Tsutsumi H, Sumiyoshi H, Chen CY. Magnetic microchains and microswimmers in an oscillating magnetic field. Biomicrofluidics. 2016;10(1):011902.

6. Shorstkii IA, Savenkov KY. Self-cleaning filter based on magnetically controlled particles. Processes and Food Production Equipment. 2017;4:20-27. (in Russian).

7. Shorstkii I, Yakovlev N. Synthesis of magnetically controlled $\mathrm{Fe} 3 \mathrm{O} 4$ composites and their enhanced microwave absorption properties. Materials Research Express. 2019;6(4):046104.

8. Shorstkii I. Magnetism in two-dimensional arrays. In: Proceedings of the International Exhibition on Mechanics, Equipment, Materials and Technologies; 2018 Mar 29-30; Krasnodar, Russian Federation. p. 435-458.

9. Jian X, Wu B, Wei Y, Dou SX, Wang X, He W, et al. Facile Synthesis of Fe3O4/GCs Composites and their Enhanced Microwave Absorption Properties. ACS Applied Materials \& Interfaces. 2016;8(9):6101-6109.

10. Du Y, Liu W, Qiang R, Wang Y, Han X, Ma J, et al. Shell Thickness-Dependent Microwave Absorption of Core-Shell Fe3O4@C Composites. ACS Applied Materials \& Interfaces. 2014;6(15):12997-13006

11. Hou Y, Yuan H, Chen H, Shen J, Li L. Controlled fabrication and microwave absorbing mechanism of hollow Fe3O4@C microspheres. Science China Chemistry. 2017;60(6):740-747. 